\title{
Integrated Care for Older People and the Implementation in the INSPIRE Care Cohort
}

\author{
C. Takeda ${ }^{1}$, S. Guyonnet' ${ }^{2}$ Y. Sumi ${ }^{3}$, B. Vellas ${ }^{2}$, I. Araujo de Carvalho ${ }^{3}$

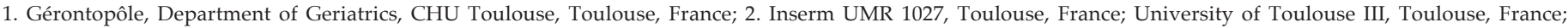

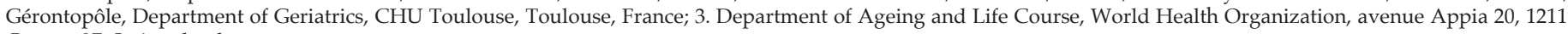
Geneva 27, Switzerland

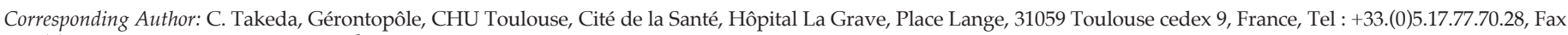
+33.(0)5.61.77.70.71, E-mail: takeda.c@chu-toulouse.fr

J Prev Alz Dis 2020;2(7):70-74

Published online March 2, 2020, http:/ / dx.doi.org/10.14283/jpad.2020.8

\begin{abstract}
BACKGROUNDS: The World Health Organization has published the Integrated Care for Older People, ICOPE handbook Guidance on person-centred assessment and pathways in primary care. This is an integrated individual care tool focused on the individual and healthy ageing. The ICOPE tool proposes step by step, a screening, a fine assessment, the development of a personalized care plan, its implementation and follow up and finally the consideration of the caregivers and community. The new Geroscience field is focusing on preventing age-related diseases, and should now investigate with the ICOPE tool the optimal maintenance of intrinsic capacity (IC) through mobility, cognition, psychology, vitality, hearing and vision. This article aims to present this new tool and to presents its innovative implementation at the Toulouse University Hospital through the INSPIRE study. We believe that the ICOPE integrated care program will also be a pragmatic way to maintain cognitive functions and detect early Alzheimer. OBJECTIVES: The main objective of the INSPIRE study is to build a Bio-resource Research Platform for Healthy Ageing gathering biological, clinical and digital resources in order to identify markers of ageing, age-related diseases and IC evolution. The study will be also testing the implementation and follow up of the ICOPE tool.

METHODS: The Inspire Platform will gather clinical data and bio-specimens from 1000 subjects in the Occitania Region, of different ages (from 30 years and over) over 10 years. Data will be collected annually. Using the ICOPE tool IC domains will be monitored every 4 months. Once IC decline is identified, participants will have a thorough clinical assessment and blood sampling to investigate the response of markers of ageing at the time of decline. The French ethic committee approved the study. RESULTS: The Inspire platform aims to develop an integrative approach to promote novel new technologies for the assessment and monitoring of functional capacities.
\end{abstract}

Key words: Integrated care, person-centred assessment, intrinsic capacity, primary care.

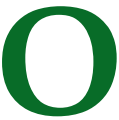
n October 1st 2019, for the International day of Older Persons, the World Health Organization (WHO) published the Integrated Care for Older People (ICOPE) handbook Guidance on personcentred assessment and pathways in primary care (1) and launched its digital app «ICOPE Handbook app» for health and social care workers (2). This article aims to present these new ICOPE tools and to show its innovative implementation at the Toulouse University Hospital through the INSPIRE study.

Currently there is an unprecedented rapid change in global demographic, so far the number of young children was higher than older people but within a few years this balance will be reversed. The number of people aged 65 and over will be greater than the number of children under 5 years old (3). This is due to the rapid increase in the proportion of older people linked in particular to longer life expectancy and decreased fertility. In 2017, there were about 962 million people aged 60 and over, comprising $13 \%$ of the global population. One in five person will be 60 years of age or older by 2050 (1). These ICOPE tools are the continuity of a work begun in 2014 by WHO, in response to the global ageing of the population, following the United Nations Sustainable Development Goals of universal coverage of care (SDG3) and in particular for older people (4). In 2015, WHO introduces «healthy ageing» defined by the development and maintenance of functional ability enabling the well-being of older people (5). Functional ability allow healthy aging through i) the response to basic needs, (ii) learning, continuing to improve and making decisions, iii) to be mobile, iv) to be able to build and maintain social relations, (v) to be able to contribute within one's family, friends and community (1). In 2017, WHO guidelines on community-level interventions to manage declines in intrinsic capacity in older people, lay the groundwork for integrated care for older people (6) based on evidencebased interventions offering 13 recommendations for health professionals. WHO defines intrinsic capacity as the composite of all physical and mental capacities and functional ability as the combination and interaction of intrinsic capacity with the person's environment (1). Trajectory of intrinsic and functional ability over the life course can be divided into three periods: a relatively stable and high capacity period, a period of capacity decline and a period of significant loss of capacity characterized by care dependency. WHO targets six 


\section{Figure 1. WHO ICOPE screening tool}

\section{WHO ICOPE SCREENING TOOL}

\begin{tabular}{|c|c|c|}
\hline $\begin{array}{l}\text { Priority conditions associated } \\
\text { with declines in intrinsic capacity }\end{array}$ & Tests & $\begin{array}{l}\text { Assess fully any domain } \\
\text { with a checked circle }\end{array}$ \\
\hline \multirow[t]{2}{*}{$\begin{array}{l}\text { COGNITIVE DECLINE } \\
\text { (Chapter 4) }\end{array}$} & $\begin{array}{l}\text { 1. Remember three words: flower, door, rice (for example) } \\
\text { 2. Orientation in time and space: What is the full date today? } \\
\text { Where are you now (home, clinic, etc)? }\end{array}$ & $\begin{array}{l}\text { Wrong to either question or } \\
\text { does not know }\end{array}$ \\
\hline & 3. Recalls the three words? & Cannot recall all three words \\
\hline $\begin{array}{l}\text { LIMITED MOBILITY } \\
\text { (Chapter 5) }\end{array}$ & $\begin{array}{l}\text { Chair rise test: Rise from chair five times without using arms. } \\
\text { Did the person complete five chair rises within } 14 \text { seconds? }\end{array}$ & No \\
\hline \multirow[t]{2}{*}{$\begin{array}{l}\text { MALNUTRITION } \\
\text { (Chapter } 6)\end{array}$} & $\begin{array}{l}\text { 1. Weight loss: Have you unintentionally lost more than } 3 \mathrm{~kg} \\
\text { over the last three months? }\end{array}$ & Yes \\
\hline & 2. Appetite loss: Have you experienced loss of appetite? & Yes \\
\hline $\begin{array}{l}\text { VISUAL IMPAIRMENT } \\
\text { (Chapter 7) }\end{array}$ & $\begin{array}{l}\text { Do you have any problems with your eyes: difficulties in } \\
\text { seeing far, reading, eye diseases or currently under } \\
\text { medical treatment (e.g. diabetes, high blood pressure)? }\end{array}$ & Yes \\
\hline \multirow{3}{*}{$\begin{array}{l}\text { HEARING LOSS } \\
\text { (Chapter 8) }\end{array}$} & Hears whispers (whisper test) or & \\
\hline & Screening audiometry result is $35 \mathrm{~dB}$ or less or & Fail \\
\hline & Passes automated app-based digits-in-noise test & \\
\hline \multirow{3}{*}{$\begin{array}{l}\text { DEPRESSIVE SYMPTOMS } \\
\text { (Chapter 9) }\end{array}$} & Over the past two weeks, have you been bothered by & Yes \\
\hline & - feeling down, depressed or hopeless? & \\
\hline & - little interest or pleasure in doing things? & Yes \\
\hline
\end{tabular}

domains of intrinsic capacity: cognition, mobility, vitality represented by nutrition, mood, vision and hearing (1). The challenge of intrinsic capacity is to identify conditions associated with declines in intrinsic capacity even before the older person becomes frail in order to delay or even reverse this decline. The conventional approach to medicine, the curative model (clinical signs, diagnosis and treatment) needs to integrate this person-centred approach to maintain the well-being of older people. Implementing the ICOPE approach and maintaining it over the life-course is a challenge for our health and social care system. WHO proposes an ICOPE approach by setting person-centred goals, supporting self-management, screening for loss in intrinsic capacity, assessing health and social care needs, supporting caregivers and developing a personalized care plan (1). There are five steps in the ICOPE approach: Step 1 is a screening in search of decline in intrinsic capacity; Step 2 is person-centered assessment in primary care. Step 3 develops a personalized care plan with a multidisciplinary team. Step 4 is the implementation of the care pathway with regular monitoring in link to specialized geriatric care. Finally, Step 5 is the integration of the caregivers and the community.

The ICOPE screening tool (1) in Step 1, proposed by WHO is a simple tool, which can be used in the community and primary care settings, after training, by health and social care workers who are not necessarily medical doctors. This tool can also be used for the followup.

The screening tool comes in the form of questions or tests to screen declines of intrinsic capacity (figure 1 ICOPE screening tool (1)). The examiner must fill in the screening tool by checking the corresponding boxes. Conducting the screening test takes about 8 minutes. These are simple and reproducible tests over time. In case of signs of decline during screening, a more detailed assessment is necessary by switching to Step 2 .

Step 2 is a more detailed, person-centred assessment. This assessment is carried out by a trained staff, but not necessarily by a medical doctor. First, you need to understand the person's life, his or her values, priorities and preferences. Assess for decline of intrinsic capacity, look for underlying diseases and assess the person's environment. This will help create personalized care plan in Step3.

Step 3 sets a goal and develops a personalized care plan in partnership with the individual, the caregivers and social workers. The objective is optimizing intrinsic capacity and functional ability in the integration of care. It is also an opportunity to monitor the progress and impact of interventions for the person. It is essential that the older person and caregiver(s) participate in the development of the goals. The personalized care plan includes self-management, advices, ICOPE interventions, management of chronic diseases and the consideration of the environment.

The Step 4 is the implementation and monitoring of the personalized care plan with referral to the specialist(s) if needed. Regular monitoring allows monitoring progress and adapting supports as needed. Addressing specialists, through defined care pathways, is essential for prompt management during acute events but also in palliative care situations. WHO stresses the importance of geriatric involvement at this level, due to the expertise in geriatric syndromes, polypharmacy and specific pathologies such as dementia. They must be intertwined with the care of the patient by assisting the primary care team.

The goal of Step 5 is to enlist the community and support caregivers. Indeed, the work of caregiver can be hard and sometimes requires the integration of interventions for the caregiver. This must be taken into 


\section{Figure 2. WHO ICOPE Pathways}
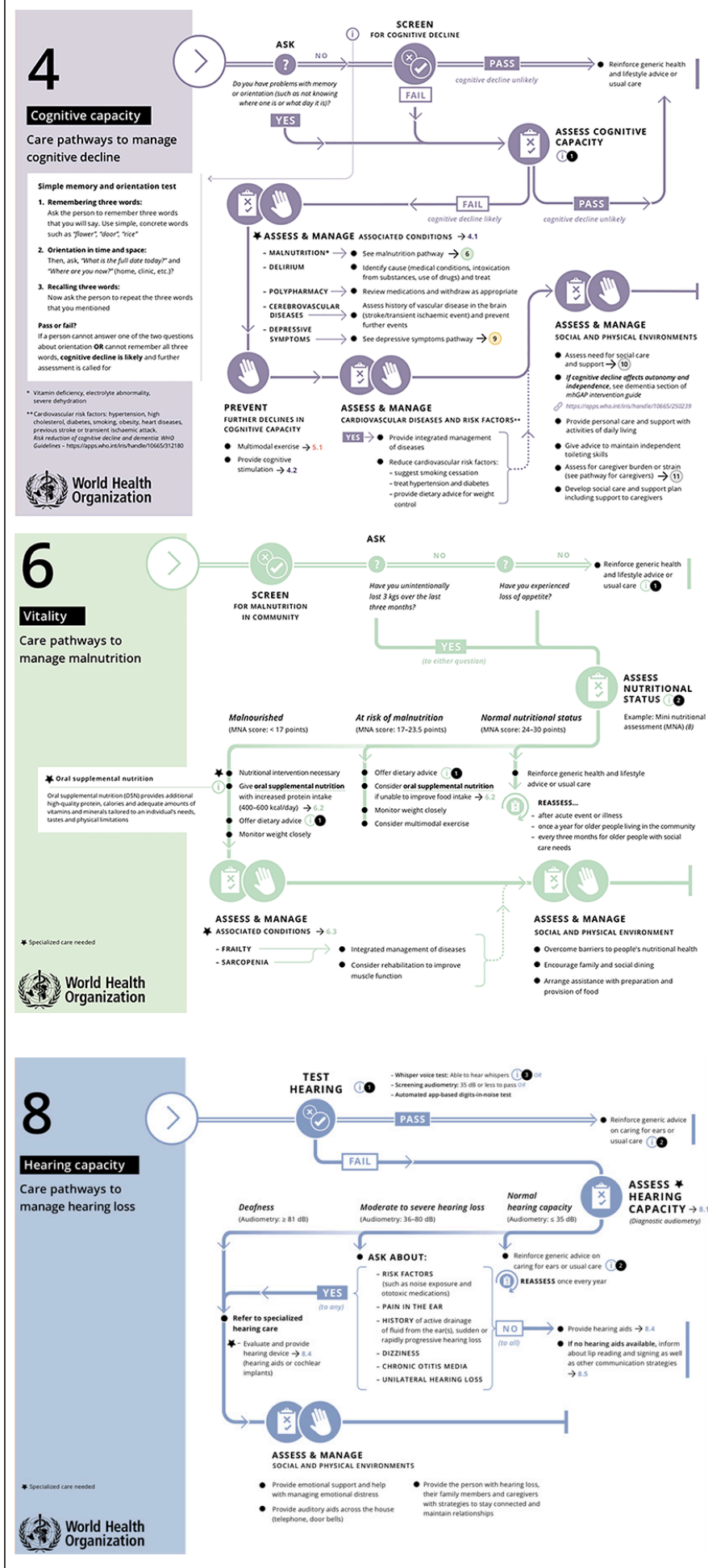

account when developing the personalized care plan (training, advice and information resources).

The ICOPE approach is based in community and primary care settings, with the aim of ensuring that it is accessible to as many people as possible and emphasizes on strong links with specialists. For each domain of intrinsic capacity, the guide offers a care pathway (1) (figure 2). The ICOPE app offers a detailed assessment of decline and a person-centred care pathway (figure 3) (2).

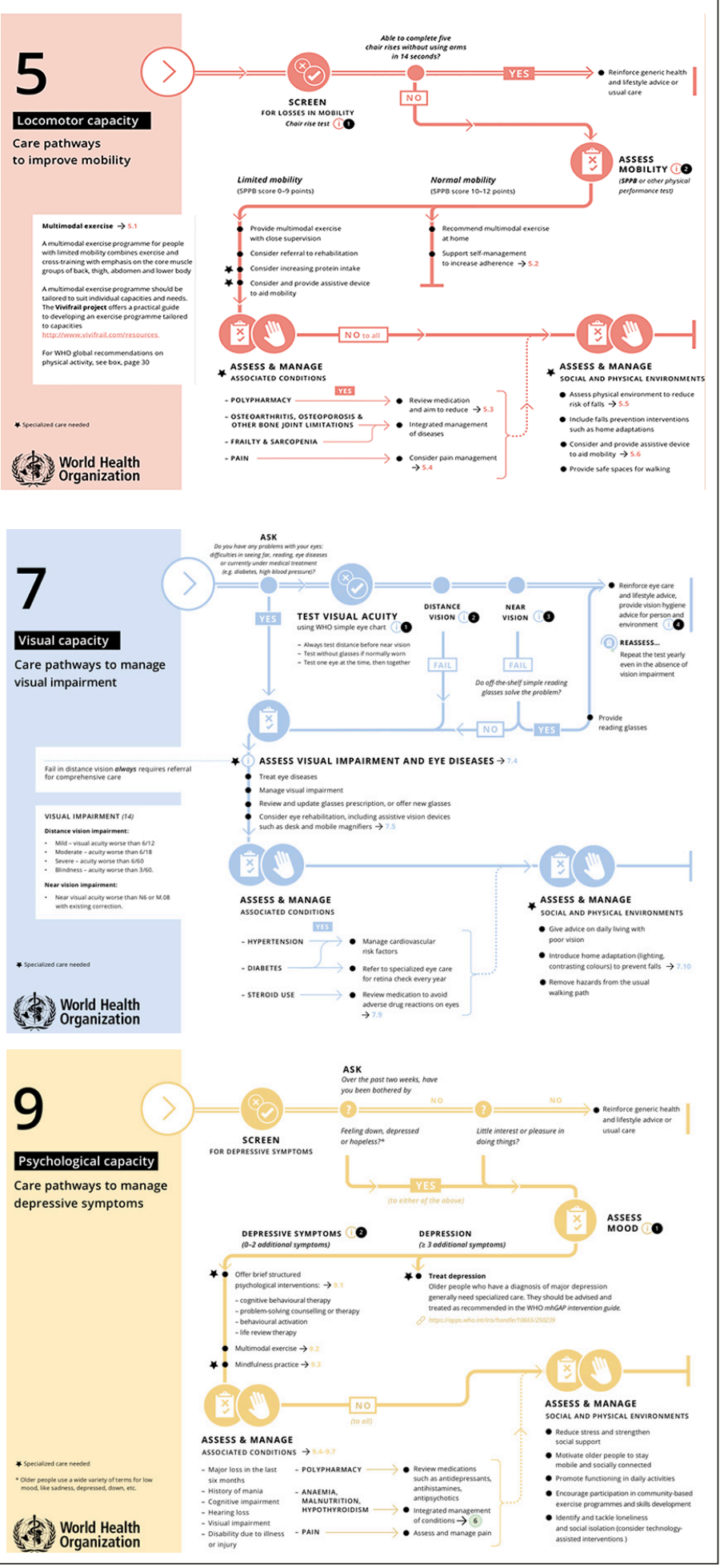

In the final part of the guide, WHO briefly addresses the issue of the implementation and long-term maintenance of the integrated approach of older people. WHO provides ICOPE implementation framework Guidance for systems and services (7). Multiple studies have attempted to implement an integrated approach to older adults, but to date there is no consensus. The project will not be viable without national support. Upstream studies are needed, to determine the feasibility of the 


\section{Figure 3. WHO ICOPE app}

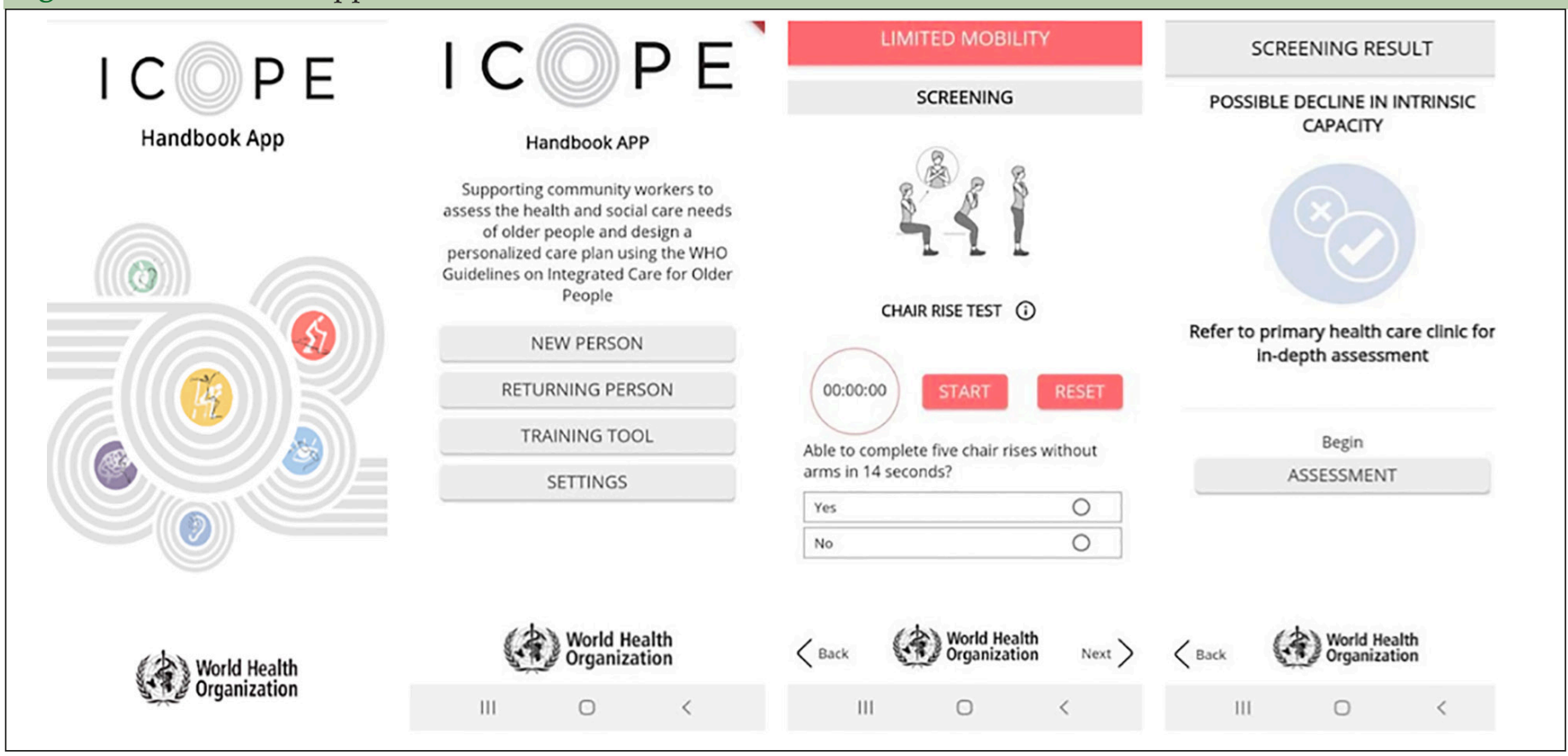

project (financial and organizational support) and the sustainability of the action (efficiency and workforce capacity). To support healthy ageing, there needs to be consistency and integration of all stakeholders, health system and social services.

At the same time, the Toulouse University Hospital is launching the INStitute for Prevention healthy agIng and medicine REjuvenative (INSPIRE) study, in align with the implementation of ICOPE in primary care in the Occitania region, by systematically applying the ICOPE screening tool every 4 months over 10 years.

The main objective of the INSPIRE study is to create an innovative research platform combining biological, clinical (including imaging data) and digital data from patient in primary care. These data will help identify biomarkers of ageing, and track the evolution of markers of the trajectory of intrinsic capacity (mobility, memory, mood, nutritional status, vision and hearing), from adulthood (30 years) to the most advanced ages (no upper age limit) and thus defining strategies for maintaining autonomy and preventing dependency.

The secondary objectives of the study are multiple:

- To create a web platform to track subjects remotely with collection of lifestyle and clinical data (especially on intrinsic capacity).

- To test the feasibility and acceptability of a smartphone and tablet application to remotely evaluate and monitor intrinsic capacity.

- Identify, using clinical, biological and digital data, markers of biological ageing that characterize systemic morphological and functional changes in the body, capable of predicting the temporal trajectory of domains of intrinsic capacity.

- To study the trajectory of different domains of intrinsic capacity over time (natural history), their interactions, predictors and the causes of their decline.

- To analyze data on the loss of intrinsic capacity, pejorative events and consumption in care, in order to define a better approach to care management.

- To study the rate of acceptability and adherence to screening, evaluation and monitoring of areas of intrinsic capacity (i.e. WHO's ICOPE program) in different clinical settings, in order to contribute to the development of health and social care pathways focused on functional status by developing a clinical cohort.

- Discover markers that could be outcomes for clinical trials in the field of Geroscience.

- Exploring the development of digital markers of ageing through the ICOPE app.

The main study focuses on biomarker research with the study of two cohorts, a human cohort called a translational cohort and an animal cohort. Biomarkers can better predict health events, better track and treat patients. The biomarkers of ageing should differentiate people of different ages, but also those with faster ageing than others. They should also be associated with changes in function or morphological changes observed during aging. Finally, these biomarkers will have to predict serious acute events, sometimes observed during ageing such as chronic diseases, cancers or addiction.

In addition to these two main study cohorts, a third cohort (care cohort) is added, enabling an epidemiological study in primary care in the Occitania region of the implementation of the ICOPE screening tool through the systematic implementation of STEP 1 every 4 months, which will be monitored by the Toulouse regional team for ageing and prevention (Equipe régionale vieillissement et prevention de la dépendance). 
A final digital cohort will only be followed by the digital ICOPE application tailored to the study's needs.

\section{Expected outcome forward}

In the event of a good response in Occitania, the Toulouse University Hospital hopes to be able to deploy this ICOPE approach at the national level. This approach is a real challenge for doctors in the context of the ageing population and the lack of a doctors in France (8). Screening for the decline of intrinsic capacity, systematic, short, achievable by all, in self-management every 4 months, would anticipate functional decline. This approach facilitates the work of the general practitioner and identifies health problems in common practice ahead of the acute event.

Acknowledgements and fundings: The Inspire Plateform is supported by grants from the Occitania Region and the European Regional Development Fund (ERDF), and co-funding by the APOC, the CTAD, and the Edenis, Korian, Pfizer, and Pierre Fabre groups. The promotion of this study is supported by the University Hospital Center of Toulouse.

Conflicts of interest: The author declares no conflict of interest.

Open Access: This article is distributed under the terms of the Creative Commons Attribution 4.0 International License (http://creativecommons.org/ licenses/by/4.0/), which permits use, duplication, adaptation, distribution and reproduction in any medium or format, as long as you give appropriate credit to the original author(s) and the source, provide a link to the Creative Commons license and indicate if changes were made.

\section{References}

1. WHO-Handbook-ICOPE.pdf [Internet]. [cité 1 oct 2019]. Disponible sur: https:/ / apps.who.int/iris/bitstream/handle/10665/326843/ WHO-FWC-ALC-19.1-eng.pdf;jsessionid=31CB3214293723D1D9 A7D2B822B92D0E?sequence $=1$

2. WHO ICOPE Handbook App- Apps on Google Play. https://play.google. $\mathrm{com} /$ store / apps / details?id=com.universaltools.icope\&hl=en. Accessed 10 November 2019. -Apps on Apple Store. https://apps.apple.com/fr/app/ who-icope-handbook-app/id1482388332. Accessed 10 December 2019.

3. global_health.pdf [Internet]. https://www.who.int/ageing/publications/ global_health.pdf. Accessed 1 October 2019.

4. SDGs ... Sustainable Development Knowledge Platform [Internet]. https:// sustainabledevelopment.un.org/sdgs. Accessed 1 October 2019.

5. Rapport mondial sur le vieillissement et la santé.pdf [Internet]. https:// apps.who.int/iris/bitstream/handle/10665/206556/9789240694842_fre. pdf?sequence $=1$. Accessed 1 October 2019 .

6. World Health Organization, Department of Ageing and Life Course Integrated care for older people: guidelines on community-level interventions to manage declines in intrinsic capacity. [Internet]. 2017 http://www.ncbi. nlm.nih.gov/books/NBK488250/. Accessed 1 October 2019.

7. Implentation framework.pdf [Internet]. https://apps.who.int/iris/bitstream/ handle / 10665/325669/9789241515993-eng.pdf?sequence=1. Accessed 2 October 2019.

8. Santé $\mathrm{M}$ des $\mathrm{S}$ et de la. Lutter contre les déserts médicaux [Internet]. Ministère des Solidarités et de la Santé. 2019 https: / / solidarites-sante.gouv.fr/systemede-sante-et-medico-social/masante2022/lutter-contre-les-deserts-medicaux / . Accessed 2 October 2019. 